rheumatology centres in Khartoum between December 2008 and September 2010 were included. Samples analysed in Uppsala for anti-CCP and IgM RF.

Results The mean age at inclusion was 48 years (range 14-80). Median disease duration was 3.8 years. ESR data was available for 113 patients, with a mean of $60.3 \mathrm{~mm} / 1 \mathrm{~h}$ (range 10-140). Mean blood haemoglobin was $12.1 \mathrm{~g} / \mathrm{L}$. On clinical examination, $26 \%(68 / 264)$ had Z deformity, 14\% (38/264) had Swan neck deformity and $9 \%$ (25/264) had Boutonniere deformity. X-rays of hands were available for 86 patients, with 49/86 (57\%) showing erosions. $40 \%$ were treated with methotrexate, $7 \%$ with sulfasalazine $3 \%$ azathioprine, $2 \%$ with leflunomide and $2 \%$ with hydroxychloroquine in monotherapy. $41(16 \%)$ were treated with steroids + DMARD monotherapy, 48 (18\%) with DMARD combinations. Three $\%$ were treated with steroids only, and 9\% with NSAIDs only.

$52 \%$ were anti-CCP2 positive and $51 \%$ were IgM RF positive, corresponding to $97.6 \%$ specificity compared to the Sudanese healthy controls. Compared to Swedish RA patients (Rönnelid et al, ARD 2012) Sudanese patients had $270 \%$ higher mean ESR (55 versus $21 \mathrm{~mm} / \mathrm{h}$; $p<0001$ ), and significantly lower age of disease onset (median 43 versus 56 years, $\mathrm{p}<0.0001$ ).

Conclusions RA as presented in an outpatient clinic in Khartoum is severe and with earlier RA onset than in Sweden. Sudanese patients show significantly higher ESR levels than Swedish patients, more Sudanese than Nigerian RA patients have radiological erosions, and the number of patients with classical hand deformities is substantial. Blood haemoglobin levels are rather well preserved. Immunological and genetic characterisation is now underway.

\section{A6.11 INTRA-ARTICULAR OVEREXPRESSION OF INTERLEUKIN-10 DIMINISHES CARTILAGE PROTEOGLYCAN DEPLETION IN STREPTOCOCCAL CELL WALL ARTHRITIS: A PROMISING CONCEPT FOR DISEASE-REGULATED GENE THERAPY}

doi:10.1136/annrheumdis-2013-203220.11

Eline A Vermeij, Mathiijs GA Broeren, Miranda B Bennink, Onno J Arntz, Wim B van den Berg, Fons AJ van de Loo. Rheumatology Research and Advanced Therapeutics, Radboud University Nijmegen Medical Centre

Background and Objectives Local gene therapy for arthritis, with the use of disease-inducible promoters, represents a promising alternative for coping with side effects of the conventional treatments. These disease-inducible promoters react to transcription factors that are released during inflammation and therefore only produce a therapeutic protein when necessary. Interleukin-10 (IL-10) could play an important regulatory role in streptococcal cell wall arthritis (SCW), and therapeutic effects are present when IL-10 was injected systemically (Lubberts et al, 1998).

In this study IL-10 was used to investigate the potential of intraarticular gene therapy in an acute model of arthritis.

Materials and Methods C57B16/N mice were injected intraarticularly in the kneejoint with lentivirus, expressing the therapeutic protein IL-10 or the luciferase reporter. Inducible promoters S100a8, Cxc11, MMP13, Saa3, IL-1b, and TNFaip6, which were selected from endogenous genes differentially regulated in the inflamed synovium of collagen-induced arthritis mice, were used to express luciferase. The constitutive PGK promoter was used to express IL-10. Arthritis was induced by injection of $25 \mu \mathrm{g} \mathrm{SCW}$ into the knee joint cavity 4 days later. At 1, 4, and 7 days after arthritis induction, mice treated with PGK-IL10 were sacrificed, and knee joints were dissected for either histological analysis, or RNA isolation for qPCR analysis. At the same timepoints, in-vivo bioluminescent imaging was performed in mice treated with the inducible promoter reporter, using the IVIS Lumina system.

Results PGK-IL10 significantly decreased proteoglycan (PG) depletion at day 4 and 7 after arthritis induction, probably by inhibiting MMPs and upregulating TIMPs. No effects on inflammation were seen histologically, but synovial IL-1, IL-6 and TNFa gene expressions were markedly decreased at day 1 . The inducible promoters all showed a different activation profile during the course of inflammation, meaning they all react differently during the disease process. The Saa3 promoter showed the highest upregulation (120 fold) and was the only promoter which showed an early peak in activation at day 1 after arthritis induction, resembling neutrophil influx.

Conclusions Effects of IL-10 were seen on PG depletion and gene expressions, therefore IL-10 can be a feasible therapeutic protein to modulate SCW arthritis. On the other hand, the Saa3 promoter seems to be the best candidate for local intra-articular gene therapy with the use of disease-inducible promoters, because it showed a high and quick upregulation during disease activity. Hence, combining the Saa3 promoter with the therapeutic protein IL-10, can be a promising combination to modulate an acute model of arthritis using disease regulated gene therapy.

\section{A6.12 LATERAL EPICONDYLE TENDON LESIONS TREATMENT WITH PLATELET GROWTH FACTORS}

doi:10.1136/annrheumdis-2013-203220.12

P González-Moreno, R Santos Sanabria, R Hernández-Sánchez, JL Marenco. Valme University Hospital, Seville, Spain

Background Chronic painful tendon disorders are common and difficult to treat. Recently, research has focused on regenerative therapies based in the injections with autologous platelet growth factors (PGRF). Two randomised clinical trials involving patients with tendinopathy receiving autologous PGRF showed conflicting results. However, better outcomes were observed among individuals randomised to PGRF injections in the trial randomising patents with lateral epicondyle tendon lesion. The potential benefit of PGRF injections in patients without response to standard therapy is not established.

Objectives To evaluate the efficacy and safety of local injections with autologous PGRF to treat epicondylitis in patients refractory to standard therapy.

Methods Patients with epicondylitis who had received standard therapy including corticosteroid local injections and NSAIDS, with or without local ice or orthesis, were included in this prospective study. Patients being treated with physiotherapy were excluded. Patients were treated with one PGRF injection per month during three months. Symptoms, side effects of injections, visual analogic scale (VAS) were recorded in every visit. Fried man test was applied to compare VAS among visits.

Results 17 patients were included, 12 (57\%) of whom were men. The median age was 52 (range: 42-61) years. 8 individuals have completed the scheduled therapy, 17 have reached 1 month. Median (RIQ) VAS were: at baseline 7 (6-9); at 1 month 6 (4-8); at 2 months 5 (3-9.5) $(p<0.001)$. An improvement in VAS was observed in 14 (82\%) patients at 1 month, and $6(75 \%)$ at 2 month. No significant side effects were observed.

Conclusions Local PGRF injections were efficacious to treat lateral epicondyle tendon lesions in patients without response to previous standard therapy. Local PGRF injections were well-tolerated.

\section{A6.13 MINOR DISCREPANCY BETWEEN BMD OF SPINE AND HIP}

doi:10.1136/annrheumdis-2013-203220.13

Poran Farahifard, Shahid Sadoughi, Mohammad Bagher Owlia, Shahid Sadoughi. University of Medical Sciences, Yazd, Iran

Background and Objectives Diagnostic discordance for osteoporosis is the observation that the T-score of an individual patient varies from one key measurement site to another, falling into two different diagnostic categories of minor and major discordances, identified by the World Health Organization (WHO) classification 
system. The aim of the present study was to assess the frequency of causes of minor discrepancy between bone densities of hip and spine.

Methods In this study, from all patients referred to bone marrow densitometery centre of Loghman from September 2008 to May 2009 in order to BMD assessment due to several causes and underwent spine and hip BMD. 171 cases with minor discrepancies were enrolled and causes of discrepancies were determined.

Results The mean age of patients was $92.69 \pm 13.13$ years. $87.78 \%$ of patients were female. The causes of minor discrepancy were respectively Osteoarthritis (45.03\%), Osteoporosis (28.65\%), Aortic Calcification, Osteoarthritis Deformans, Syndesmophytes and DISH each with $2.92 \%$. These causes in female gender were Osteoarthritis (48.67\%), Osteoporosis (27.33\%), Aortic Calcification and Syndesmophytes with 3.33\%; and in male they were Osteoarthritis (38.1\%), Osteoporosis and DISH each with $19.05 \%$.

Conclusions The minor discrepancy in T-score is a common finding which can result diagnostic problems in densitometry analysis. The results of the present study show that the most common causes of minor discrepancy were Osteoarthritis, Osteoporosis, Aortic Calcification, Osteoarthritis Deformans, Syndesmophytes and DISH.

\section{A6.14 mTOR DIRECTED MESENCHYMAL TISSUE RESPONSE TO INFLAMMATION IN ARTHRITIS}

doi:10.1136/annrheumdis-2013-203220.14

1,2T Karonitsch, ${ }^{2} \mathrm{~K}$ Dalwigk, ${ }^{3} \mathrm{M}$ Glehr, ${ }^{2 B}$ Niederreiter, ${ }^{2} \mathrm{CW}$ Steiner, ${ }^{2} J S$ Smolen, ${ }^{2} \mathrm{HP}$ Kiener, 'G Superti-Furga. ${ }^{1} \mathrm{CeMM}$ Research Center for Molecular Medicine of the Austrian Academy of Sciences; 'Division of Rheumatolotgy, Department of Medicine 3, Medical University of Vienna; ${ }^{3}$ Department of Orthopaedic Surgery, Medical University of Graz

Background Accumulating evidence supports the concept that fibroblast-like synoviocytes (FLS) actively participate in the destructive, inflammatory process of rheumatoid synovitis. Thus, FLS frame a synovial microenvironment that augments and perpetuates synovial inflammation. Moreover, FLS, together with macrophages form an aggressive mass of cells ("pannus"), which invades and destroys the articular cartilage. The mechanistic target of rapamycin (mTOR) is best known for coupling energy and nutrient abundance to the execution of essential cellular processes, including cell growth and cell survival. More recent data indicate that mTOR directs the cellular response to inflammatory stimuli in cells of the immune system. It remains elusive, however, whether or not this also applies to mesenchymal cells, such as FLS in the context of rheumatoid synovitis.

Materials and Methods In order to assess mTOR activity by immunhistochemistry (IHC) as well as western blotting (WB), phosphospecific antibodies against mTOR (IHC) and mTOR substrates, including 4E-BP (IHC), AKT (WB), S6K1 (WB), and S6 (IHC) were used. To determine the functional significance of mTOR activity in FLS, Torin-1, a well defined, specific inhibitor of mTOR, was used. To establish a role for mTOR in the mesencyhmal, inflammatory tissue response, we used a previously described simplified 3-D model of the synovial tissue. IL-6 and IL- 8 levels in the supernatants of 3-D cultures were measured by ELISA.

Results mTOR, 4E-BP and S6 were found to be phosphorylated in RA synovial tissues. These activated phospho-proteins were preferentially expressed in FLS, most prominently in the hyperplasic synovial lining layer. In-vitro, TNF stimulation of FLS resulted in the phosphorylation of AKT and S6K1, indicating that TNF activates the mTOR pathway in FLS. Stimulation of the $3 \mathrm{D}$ cultures with TNF resulted in hyperplasia of the lining layer at the surface of the spheres. Strikingly, treatment with Torin-1, prevented TNF induced lining layer hyperplasia. Unexpectedly, the combined treatment of 3-D cultures with TNF and Torin-1 resulted in increased production of IL- 6 as well as IL-8 when compared to cultures that were solely exposed to TNF.

Conclusions These studies provide insight into the regulatory circuits that determine the synovial mesenchymal tissue response to inflammation and suggest a multifaceted role for mTOR in arthritis

\section{A6.15 REFRACTORY CHRONIC ERYTHEMA NODOSUM AND TREATMENT WITH ANTI TNF}

doi:10.1136/annrheumdis-2013-203220.15

J Uceda, R Hernández, L Mayordomo, JL Marenco. Rheumatology Department, Valme University Hospital, Seville, Spain

Introduction Erythema nodosum septal panniculitis without vasculitis, characterised by acute episodes of inflammatory and painful subcutaneous nodules affecting, in most cases, the lower extremities. Remission of lesions occurs within 1-6 weeks without scarring or residual atrophy. There are, however, some cases which become chronic or reoccur. In $50 \%$ of cases we find an underlying cause. Treatment of acute outbreak involves rest and NSAIDs. The chronic or recurrent cases are treated with oral potassium iodide, corticosteroids, colchicine, hydroxychloroquine or immunosuppressive agents.

Objectives Description of the cases of refractory chronic erythema nodosum and review of the literature.

Methods Selection of patients with refractory chronic erythema nodosum undergoing treatment with anti TNF in the Rheumatology unit from 2000 to 2010. Literature search using PubMed with keywords erythema nodosum and Adalimumab, Etanercept, Infliximab.

Results See table 1.

Conclusions In our sample, all cases have responded favourably to treatment with anti TNF. No adverse events were observed, except the occurrence of cutaneous psoriasis in one patients after

Abstract A6.15 Table

\begin{tabular}{|c|c|c|c|c|}
\hline Patient & 1 & 2 & 3 & 4 \\
\hline Sex & Female & Female & Male & Female \\
\hline Age & 38 & 32 & 40 & 43 \\
\hline Diagnosis & Behçet's disease & Behçet's disease & Sarcoidosis & Psoriatic arthritis \\
\hline Pre-treatment & $\begin{array}{l}\text { Prednisone } 30 \mathrm{mg} / 24 \mathrm{~h} \\
\text { Azathioprine } 100 \mathrm{mg} / 24 \mathrm{~h} \\
\text { NAID maximun dosage }\end{array}$ & $\begin{array}{l}\text { Prednisone } 30 \mathrm{mg} / 24 \mathrm{~h} \\
\text { Azathioprine } 100 \mathrm{mg} / 24 \mathrm{~h}\end{array}$ & $\begin{array}{l}\text { Prednisone } 30 \mathrm{mg} / 24 \mathrm{~h} \\
\text { Azathioprine } 100 \mathrm{mg} / 24 \mathrm{~h} \\
\text { NAID maximun dosage }\end{array}$ & $\begin{array}{l}\text { Prednisone } 30 \mathrm{mg} / 24 \mathrm{~h} \\
\text { Azathioprine } 100 \mathrm{mg} / 24 \mathrm{~h} \\
\text { NAID maximum dosage }\end{array}$ \\
\hline Anti TNF & $\begin{array}{l}\text { Infliximab } 300 \mathrm{mg} \text { every } 8 \text { weeks } \\
\text { Etanercept } 50 \mathrm{mg} \text { weekly }\end{array}$ & Infliximab 300 mg every 8 weeks & Infliximab 375 mg every 8 weeks & Infliximab 300 mg every 8 weeks \\
\hline Evolution & $\begin{array}{l}\text { Psoriasis Outbreak with infliximab. } \\
\text { Improvement of erythema nodosum with both anti TNF } \\
\text { treatments a month after start of treatment }\end{array}$ & $\begin{array}{l}\text { Principio del formulario } \\
\text { Improvement a month after start of treatment with } \\
\text { resolution in the second dose }\end{array}$ & $\begin{array}{l}\text { Asymptomatic after the first } \\
\text { infusion }\end{array}$ & $\begin{array}{l}\text { Resolution of lesions after the } \\
\text { 4th infusion of infliximab }\end{array}$ \\
\hline
\end{tabular}

